adherence and other aspects of basic management, as far as possible. We retrospectively evaluated 166 patients (median age at referral 11.7 years $[4 ; 17]$; $61 \%$ males). SAFS $(n=76)$ was defined as specific IgE (spIgE) or skin prick test (SPT) positivity to any of Aspergillus fumigatus, Alternaria alternata or Cladosporium herbarum. Non-sensitised patients $(n=90)$ had negative spIgE and SPT to all fungal allergens tested. Age of onset, atopy, symptoms (asthma control test), medication usage, lung function and airway inflammation were assessed. Results More boys had SAFS (57/76 (75\%) vs 43/90 (48\%), $\mathrm{p}<0.001$ ). Children with SAFS had earlier onset of asthma (median 0.5 years $[0 ; 12.5]$ vs $1.5[0 ; 12.5], \mathrm{p}=0.006)$, higher total serum $\operatorname{IgE}$ (637 IU/ml [12;6737] vs 177 [1;10 881], $\mathrm{p}=0.002)$ and higher sum of inhalant allergen SPT and spIgE [Allergy 2007;62:1379;86] $(16 \mathrm{~mm}$ [0;38] vs $9 \mathrm{~mm}[0 ; 36], \mathrm{p}<0.001$, and $78 \mathrm{IU} / \mathrm{ml}[0 ; 400]$ vs $19 \mathrm{IU} / \mathrm{ml}$ [0;243], $p=0.02$, respectively). Children with SAFS had a lower $\mathrm{FEV}_{1}$ (1.4 L [0.5;3.8], vs 1.9 [0.7;4.3], $\mathrm{p}=0.008)$ and lower FVC (2.3 L [0.6;4] vs $2.6[0.8 ; 5.5], \mathrm{p}=0.045)$. Bronchodilator reversibility was more frequent in SAFS ( $\mathrm{n}=59 / 73$ (81\%) vs 42/81 (52\%), $\mathrm{p}<0.001)$. Maintenance oral steroids were more frequently prescribed in SAFS $(\mathrm{n}=18 / 76(24 \%)$ vs $8 / 88(9 \%), \mathrm{p}=0.02)$. Symptoms and airway inflammation (assessed in sputum, bronchoalveolar lavage and endobronchial biopsy) were similar in children with and without fungal sensitisation.

Conclusions Children with STRA and fungal sensitisation had lower lung function, earlier asthma onset, more atopy and more bronchodilator responsiveness. There is a need for a randomised controlled trial of antifungal therapy in paediatric SAFS.

\section{S83 REPEATABILITY AND INTER-RELATIONSHIPS OF SMALL AIRWAY BIOMARKERS}

doi:10.1136/thoraxjnl-2011-201054b.83

\begin{abstract}
${ }^{1} \mathrm{~S}$ Gonem, ${ }^{2}$ Ball, ${ }^{1} \mathrm{~S}$ Corkill, ${ }^{1} \mathrm{D}$ Desai, ${ }^{1} \mathrm{~A}$ Singapuri, ${ }^{3} \mathrm{P}$ Gustafsson, ${ }^{2} \mathrm{~J}$ Owers-Bradley, ${ }^{1} \mathrm{C}$ Brightling, 'S Siddiqui. ' Glenfield Hospital, Leicester, UK; ${ }^{2}$ University of Nottingham, Nottingham, UK; ${ }^{3}$ Queen Silvia Children's Hospital, Göteborg, Sweden
\end{abstract}

Introduction and objectives There is evidence that the small airways may have an important role in asthma, and a number of physiological techniques have been developed to assess small airway dysfunction. We aimed to determine the within-visit and betweenvisit repeatability of putative small airway biomarkers, and explore the inter-relationships between them.

Methods We recruited 17 patients with moderate asthma (GINA 3/ 4), twelve patients with severe asthma (GINA 5) and fifteen healthy control subjects. Participants attended baseline, 2-week and

Abstract S83 Table 1 Principal components analysis of small airway biomarkers

\begin{tabular}{lcccc}
\hline & Component 1 & Component 2 & Component 3 & Component 4 \\
\hline FEV $_{1}$ & 0.569 & -0.461 & -0.298 & -0.198 \\
$\mathrm{FVC}$ & 0.027 & 0.008 & $-\mathbf{0 . 8 6 2}$ & 0.178 \\
$\mathrm{MEF}$ & $\mathbf{0 . 7 8 5}$ & -0.107 & -0.183 & -0.444 \\
$\mathrm{RV} / \mathrm{TLC}$ & 0.059 & 0.240 & $\mathbf{0 . 8 3 9}$ & 0.248 \\
$\mathrm{Kco}$ (\% pred) & 0.098 & -0.125 & -0.068 & $-\mathbf{0 . 7 5 4}$ \\
$\mathrm{S}_{\text {cond }}$ & 0.410 & 0.353 & 0.530 & 0.087 \\
$\mathrm{~S}_{\text {acin }}$ & -0.370 & 0.457 & -0.250 & 0.586 \\
$\mathrm{R}-\mathrm{R} 20$ & 0.073 & $\mathbf{0 . 9 5 9}$ & 0.151 & -0.038 \\
$\mathrm{AX}$ & -0.079 & $\mathbf{0 . 9 5 5}$ & 0.140 & 0.131 \\
$\mathrm{FeNO}_{200}$ & $\mathbf{0 . 8 5 5}$ & 0.039 & 0.129 & 0.123 \\
$\mathrm{NO}_{\text {alv }}$ & $\mathbf{0 . 9 0 2}$ & 0.026 & 0.219 & 0.168 \\
$\mathrm{ADC}$ & 0.346 & -0.119 & 0.040 & $\mathbf{0 . 7 9 7}$ \\
\hline
\end{tabular}

Variables that load strongly (loading score $>0.75$ ) onto each factor are highlighted. $\mathrm{FEV}_{1}$, forced expiratory volume in one second; FVC, forced vital capacity; MEF, midexpiratory flow; RV, residual volume; TLC, total lung capacity; Kco, carbon monoxide transfer coefficient; $\mathrm{AX}$, reactance area; $\mathrm{FeNO}_{200}$, fractional exhaled nitric oxide at $200 \mathrm{ml} / \mathrm{s}$ flow; $\mathrm{NO}_{\mathrm{alv}}$, alveolar nitric oxide; ADC, apparent diffusion coefficient. 3-month visits. At each visit, participants undertook standard pulmonary function tests, multiple breath washout (MBW), impulse oscillometry (IOS) and measurement of exhaled nitric oxide at multiple flow rates. Five healthy subjects and 10 patients with asthma also undertook hyperpolarised helium-3 MRI at the baseline and 3-month visits. This was used to calculate the apparent diffusion coefficient (ADC), a measure of alveolar airspace size.

Results $\mathrm{S}_{\text {acin }}$, a MBW marker of acinar airspace disease, showed excellent repeatability in patients with asthma, with intraclass correlation coefficients (ICC) of $0.914,0.897$ and 0.879 for withinvisit, 2 -week and 3-month repeatability respectively. The IOS small airway markers R5-R20 and AX displayed similarly good repeatability (0.966 [within-visit], 0.905 [2-week] and 0.844 [3-month] for R5-R20, and 0.977 [within-visit], 0.875 [2-week] and 0.855 [3-month] for $\mathrm{AX}$ ). The 3 -month repeatability for $\mathrm{ADC}$ was 0.682 . Principal components analysis was used to explore the inter-relationships between the small airway biomarkers. Four components were extracted, as shown in Abstract S83 table 1. The highest loading variables on each component were $\mathrm{FeNO}_{200}, \mathrm{NO}_{\mathrm{alv}}$ and $\mathrm{MEF}$ on component 1, R5-R20 and AX on component 2, FVC and RV/TLC on component 3 , and $\mathrm{Kco}$ (\% predicted) and $\mathrm{ADC}$ on component 4 . Thus, components $1-4$ corresponded broadly to the concepts of "airway inflammation", "frequency dependence of small airway resistance and elastance", "air trapping" and "alveolar disease" respectively.

Conclusions The putative small airway markers under investigation are robust and repeatable. Principal components analysis has revealed that the information obtained from multiple tests of airway function may be condensed down to four primary concepts, and that there is significant redundancy among the measurements.

\section{S84 CAN CLINICIANS ACCURATELY PREDICT NON-ADHERENCE TO MEDICATION IN PATIENTS WITH DIFFICULT ASTHMA? A COMPARISON BETWEEN CLINICAL JUDGEMENT AND PRESCRIPTION ISSUE DATA}

doi:10.1136/thoraxjnl-2011-201054b.84

${ }^{1} \mathrm{~A} C$ Murphy, ${ }^{2} \mathrm{~S}$ Belingheri, ${ }^{3} \mathrm{C}$ E Brightling, ${ }^{3} \mathrm{~A}$ J Wardlaw, ${ }^{3} \mathrm{D}$ Pavord, ${ }^{3} \mathrm{P}$ Bradding, ${ }^{3} \mathrm{R}$ H Green. ${ }^{1}$ University Hospitals of Leicester NHS Trust, Leicester, UK; ${ }^{2}$ School of Pharmacy, Grenoble, France; ${ }^{3}$ Institute of Lung Health, Leicester, UK

Background It is increasingly recognised that sub-optimal adherence to prescribed medication regimes is common in patients with difficult asthma and may be associated with adverse clinical outcomes. Simple reliable methods of measuring non-adherence have not been developed and clinicians' judgement is often relied upon as the only assessment of adherence. We have previously shown that checking prescription data are a useful method of investigating non-adherence in this patient population. We aimed to determine how well a clinician's judgement of adherence correlated to adherence as measured by prescription issue data.

Methods Adult asthma patients attending a difficult asthma clinic during July/August 2010 were included. GP retrospective prescription issue data and hospital dispensing data for asthma medications, patient demographics and clinical outcome data were collated. The medication adherence score was calculated as the number of doses issued/number of doses prescribed $\times 100$ for a mean duration of 12 months. Clinicians with an expertise in the management of difficult asthma were asked to stratify each patient according to their perceived adherence, classified as adherent $(=80 \%$ adherence score; partially adherent $50 \%-79 \%$; non-adherent $<50 \%$ ). Agreement between clinician judgement and adherence score was analysed using a weighted $\kappa$ coefficient. Logistic regression was performed to determine whether any clinical features could predict better agreement.

Results Data from 63 consecutive patients was included in the analysis (41 female, median (range) age 51.2(61.3)). Clinicians suspected non-adherence in $11 / 63(17.4 \%)$ of patients, partial 
non-adherence in 23/63 (36.5\%) and good adherence in 29/63 (46\%). Agreement between clinician assessment and prescription issue data were seen in only $23 / 63$ (36.5\%) of cases and overall agreement using a weighted $\kappa$ coefficient was poor (weighted agreement $63.5 \%$, expected agreement $58.8 \%, \kappa 0.11$, SE $0.1, z=1.16, p=0.12)$. There was no relationship between patients' age, gender, Juniper asthma control score, prescribed inhaled corticosteroid dose or $\mathrm{FEV}_{1}$ percent predicted and the chances of agreement or disagreement between the two methods.

Conclusions Clinical judgement alone appears to be a poor predictor of adherence to medication in patients with difficult asthma. The assessment of non-adherence requires objective measurements. Prescription issue data are one such measurement; but further work is required.

\section{Lung cancer: advances in treatment}

\section{S85 BRITISH THORACIC ONCOLOGY GROUP TRIAL, BTOG2: RANDOMISED PHASE III CLINICAL TRIAL OF GEMCITABINE COMBINED WITH CISPLATIN $50 \mathrm{MG} / \mathrm{M}^{2}$ (GC50) VS CISPLATIN $80 \mathrm{MG} / \mathrm{M}^{2}$ (GC80) VS CARBOPLATIN AUC 6 (GCB6) IN ADVANCED NSCLC}

\section{doi:10.1136/thoraxjnl-2011-201054b.85}

${ }^{1} \mathrm{D}$ Ferry, ${ }^{2} \mathrm{~L} J$ Billingham, ${ }^{2} \mathrm{H}$ W Jarrett, ${ }^{3} \mathrm{D}$ Dunlop, ${ }^{4} \mathrm{~J}$ Thompson, ${ }^{5} \mathrm{M}$ Kumar, ${ }^{6} \mathrm{G}$ Skailes, ${ }^{7} \mathrm{M}$ Nicolson, ${ }^{8} \mathrm{R}$ Shah, ${ }^{9} \mathrm{P}$ Leonard, ${ }^{10} \mathrm{~A}$ Chetiyawardana, ${ }^{11} \mathrm{P}$ Wells, ${ }^{12} \mathrm{C}$ Lewanski, ${ }^{13} \mathrm{P}$ Woll, ${ }^{14} \mathrm{~B}$ Crosse, ${ }^{2} \mathrm{M}$ Hill, ${ }^{2} \mathrm{~S}$ Pirrie, ${ }^{15} \mathrm{~K} \mathrm{~J}$ O'Byrne. ${ }^{1} \mathrm{New}$ Cross Hospital, Wolverhampton, UK; ${ }^{2}$ University of Birmingham, Birmingham, UK; ${ }^{3}$ Beaston West of Scotland Cancer Centre, Glasgow, UK; ${ }^{4}$ Birmingham Heartlands Hospital, Birmingham, UK; ${ }^{5}$ Derby Royal Hospital, Derby, UK; ${ }^{6}$ Royal Preston Hospital, Preston, UK: ${ }^{7}$ Aberdeen Royal Infirmary, Aberdeen, UK; ${ }^{8}$ Kent Oncology Centre, Kent, UK; ${ }^{9}$ Whittington Hospital, London, UK; ${ }^{10}$ Manor Hospital, Walsall, UK; ${ }^{11}$ Whipps Cross Hospital, London, UK; ${ }^{12}$ Imperial College Healthcare, London, UK; ${ }^{13}$ University of Sheffield, Sheffield, UK; ${ }^{14}$ Huddersfield Royal Infirmary, Huddersfield, UK; ${ }^{15}$ St James' Hospital, Dublin, Ireland

Background Platins are considered key drugs in treating advanced NSCLC. Carboplatin has been reported as inferior to cisplatin in meta-analyses while the optimal dose of cisplatin is unclear.

Methods Eligibility was by histologically proven NSCLC, PS 0-2, stage IIIB/IV disease and a GFR of $>60 \mathrm{ml} / \mathrm{min}$, using the Wright equation. Treatment was gemcitabine $\left(1250 \mathrm{mg} / \mathrm{m}^{2}\right)$ combined with cisplatin $50 \mathrm{mg} / \mathrm{m}^{2}$, cisplatin $80 \mathrm{mg} / \mathrm{m}^{2}$ or carboplatin AUC 6 , for up to four cycles. Carboplatin dosing was calculated using the Calvert equation. At the time of analysis 1223 deaths had been reported, allowing analysis according to the statistical plan.

Results 1363 patients were randomised between April 2005 and November 2009. Trial arms were well balanced for PS, stage and age. Median age was 63, 32\% were PS0, 60\% PS1 and 8\% PS2. The median delivered dose intensities (planned=100\%) for platinum were GC50 99\%, GC80 96\% and GCb6 87\%, for gemcitabine were $95 \%, 88 \%$ and $80 \%$ respectively. During treatment the proportion of patients experiencing at least one grade 3/4 AE were; GC50 27\%, GC80 41\% and GCb6 57\%. At analysis 140 patients were alive and median follow-up was 21 months. Response rates were significantly different between arms; GC50 23\%, GC80 33\% and GCb6 28\% $(\mathrm{p}=0.01)$. Median survival was: GC50 8.3 months, GC80 9.5 months and GCb6 10.0 months, with the GC50 arm statistically identified as differing from the other two. For subsequent primary comparisons of non-inferiority of GC50 v GC80 ( $\mathrm{HR}=1.11)$ and GCb6 v GC80 ( $\mathrm{HR}=0.96)$, the $95 \%$ CI for the cisplatin dose comparison $(0.96,1.27)$ did not exclude the pre-defined inferiority region of $\mathrm{HR}>1.2$ whereas the $95 \%$ CI for the GCb6 v GC80 comparison $(0.84,1.10)$ fell well below this inferiority region.

Conclusion In advanced NSCLC the dose of cisplatin is important with GC50 giving the poorest outcome in terms of overall survival and response rate. GCb6 is not inferior to GC80 thus, in combination with gemcitabine, and in relation to survival time, carboplatin is clinically equivalent to that of cisplatin but other factors, such as quality of life, may influence treatment choice.

\section{S86 QUALITY OF LIFE IN ADVANCED NON-SMALL CELL LUNG CANCER, EFFECTS OF CISPLATIN DOSE AND CARBOPLATIN IN COMBINATION WITH GEMCITABINE: RESULTS FROM BTOG2, A BRITISH THORACIC ONCOLOGY GROUP PHASE III TRIAL IN 1363 PATIENTS}

doi:10.1136/thoraxjnl-2011-201054b.86

${ }^{1} \mathrm{~L} J$ Billingham, ${ }^{1} \mathrm{P}$ Gaunt, ${ }^{1} \mathrm{H}$ W Jarrett, ${ }^{2} \mathrm{D}$ Dunlop, ${ }^{3} \mathrm{~J}$ Thompson, ${ }^{4} \mathrm{~K} J$ O'Byrne, ${ }^{5} \mathrm{M}$ Kumar, ${ }^{6} \mathrm{G}$ Skailes, ${ }^{7} \mathrm{M}$ Nicolson, ${ }^{8} \mathrm{R}$ Shah, ${ }^{9} \mathrm{P}$ Leonard, ${ }^{10} \mathrm{~A}$ Chetiyawardana, ${ }^{11} \mathrm{P}$ Wells, ${ }^{12} \mathrm{C}$ Lewanski, ${ }^{13} \mathrm{P}$ Woll, ${ }^{14} \mathrm{~B}$ Crosse, ${ }^{1} \mathrm{M}$ Hill, ${ }^{15} \mathrm{D}$ Ferry. ${ }^{1}$ University of Birmingham, Birmingham, UK; ${ }^{2}$ Beaston West of Scotland Cancer Centre, Glasgow, UK; ${ }^{3}$ Birmingham Heartlands Hospital, Birmingham, UK; ${ }^{4}$ St James' Hospital, Dublin, Ireland; ${ }^{5}$ Derby Royal Hospital, Derby, UK; ${ }^{6}$ Royal Preston Hospital, Preston, UK; ${ }^{7}$ Aberdeen Royal Infirmary, Aberdeen, UK; ${ }^{8}$ Kent Oncology Centre, Kent, UK; ${ }^{9}$ Whittington Hospital, London, UK; ${ }^{10}$ Manor Hospital, Walsall, UK; ${ }^{11}$ Whipps Cross Hospital, London, UK; ${ }^{12}$ Imperial College Healthcare, London, UK; ${ }^{13}$ University of Sheffield Sheffield, UK; ${ }^{14}$ Huddersfield Royal Infirmary, Huddersfield, UK; ${ }^{15}$ New Cross Hospital, Wolverhampton, UK

Background The standard of care for advanced NSCLC is platinumbased chemotherapy but the optimal dose of cisplatin and comparison with carboplatin is uncertain. With median survival at 8-12 months, the impact of such treatment choices on patients' quality of life $(\mathrm{QoL})$ is important. The BTOG2 trial is a large phase III randomised trial comparing three treatment arms: gemcitabine $(1250 \mathrm{mg} /$ $\mathrm{m}^{2}$ day 1 and day 8 ) with either cisplatin $80 \mathrm{mg} / \mathrm{m}^{2}$ (GC80), cisplatin $50 \mathrm{mg} / \mathrm{m}^{2}$ (GC50) or carboplatin AUC6 (GCb6). The trial was innovative in aiming to collect QoL data on all trial patients and is the largest study to date addressing this issue in NSCLC.

Methods QoL was measured at each cycle of chemotherapy and each follow-up visit using standard, validated questionnaires: EORTC OLQ-C30, LC13 and EQ-5D.

Results More than 8000 questionnaires were returned from 1363 randomised patients with compliance around $90 \%$ during the treatment period. At pre-randomisation, the mean global heath status score and EQ-5D utility score were $62 \%$ and 0.66 . On initiation of treatment, patients in all three treatment arms had improved pain, cough, haemoptysis, insomnia, appetite loss and emotional functioning with associated improvements in global measures of QoL but these benefits generally fell away after completion of chemotherapy $(12+$ weeks). GC50 performed better in terms of the functioning scores and in terms of fatigue, nausea and vomiting while GCb6 performed worst for dyspnoea. All treatments had a deleterious effect on peripheral neuropathy with the post-treatment toxicity momentum markedly worse for GC80. Mean quality-adjusted life months were 6.1 on GC80, 5.6 on GC50 and 6.1 on GCb6.

Conclusion Although higher doses of cisplatin $\left(80 \mathrm{mg} / \mathrm{m}^{2}\right)$ are thought detrimental to $\mathrm{QoL}$ compared to $50 \mathrm{mg} / \mathrm{m}^{2}$ we found minimal differences but a noteworthy problem in delayed neuropathy. Also, the belief that carboplatin produces superior QoL compared to cisplatin at either dose is not obvious. Importantly carboplatin treatment may not palliate dysponea as well as cisplatin. Adjusting for QoL does not change the conclusions from the primary survival analysis.

\section{S87 DELIVERED DOSE INTENSITY OF GEMCITABINE $1250 \mathrm{MG} / \mathrm{M}^{2}$ WITH CISPLATIN AT $80 \mathrm{MG} / \mathrm{M}^{2}$ (GC80) AND $50 \mathrm{MG} / \mathrm{M}^{2}$ (GC50) AND CARBOPLATIN AUC 6 (GCB6) IN A PHASE III TRIAL OF ADVANCED NON-SMALL CELL LUNG CANCER (NSCLC): CORRELATIONS WITH CLINICAL OUTCOMES}

doi:10.1136/thoraxjn-2011-201054b.87

${ }^{1} \mathrm{D}$ Dunlop, ${ }^{2} \mathrm{D}$ Ferry, ${ }^{3} \mathrm{H}$ W Jarrett, ${ }^{3} \mathrm{~L} J$ Billingham, ${ }^{4} \mathrm{~J}$ Thompson, ${ }^{5} \mathrm{M}$ Kumar ${ }^{6} \mathrm{G}$ Skailes, ${ }^{7} \mathrm{M}$ Nicolson, ${ }^{8} \mathrm{R}$ Shah, ${ }^{9} \mathrm{P}$ Leonard, ${ }^{10} \mathrm{~A}$ Chetiyawardana, ${ }^{11} \mathrm{P}$ Wells, 